\title{
Wave-breaking condition of space-charge waves in a relativistic electron beam
}

\author{
Abraham C.- L. Chian \\ Institute for Space Research, P.O. Box 515, 12201 São José dos Campos, São Paulo, Brazil
}

(Received 20 April 1988; revised manuscript received 12 September 1988)

\begin{abstract}
Wave-breaking conditions, in terms of the electron velocity or the maximum wave amplitude, of space-charge waves in a relativistic electron beam are obtained. The discussion includes both negative-energy and positive-energy modes as well as wave propagation parallel and antiparallel to the electron beam.
\end{abstract}

Space-charge waves resulting from the longitudinal bunching of space charges in a relativistic electron beam are of primary importance for the operation of highpower sources of coherent radiation and high-energy collective ion accelerators. For example, in conventional Raman free-electron lasers, ${ }^{1-3}$ an instability is excited when the negative-energy space-charge wave interacts with the positive-energy electromagnetic wave leading to the growth of the scattered radiation; in converging guide ion accelerators, ${ }^{4-6}$ ions are first trapped in the potential troughs of negative-energy space-charge waves and then accelerated to high energy by increasing the phase velocity of the space-charge waves to near the electron-beam velocity.

As the space-charge wave reaches large amplitudes, higher harmonics of the fundamental wave oscillation are generated which leads to the nonlinear steepening of the wave. The steepening of the space-charge wave proceeds until the wave attains a critical amplitude beyond which wave-breaking occurs. The concept of wave-breaking was first introduced in plasma physics to describe the nonlinear saturation of electron plasma waves. It was shown that, for nonrelativistic nonlinear electron plasma waves, ${ }^{7}$ the wave-breaking condition is $e E_{\max } / m \omega_{p} v_{\mathrm{ph}}=1$, where the electron plasma frequency $\omega_{p}=\left(n_{0} e^{2} / m \epsilon_{0}\right)^{1 / 2}$ and the wave phase velocity $v_{\mathrm{ph}}=\omega / k$; whereas, for relativistic nonlinear electron plasma waves, ${ }^{8-11}$ the wave-breaking condition is

$$
e E_{\max } / m \omega_{p} c=\sqrt{2}\left(\gamma_{\mathrm{ph}}-1\right)^{1 / 2},
$$

where

$$
\gamma_{\mathrm{ph}}=\left(1-v_{\mathrm{ph}}^{2} / c^{2}\right)^{-1 / 2} .
$$

The aim of this paper is to determine the wavebreaking condition of space-charge waves in a relativistic electron beam. The discussion is generalized to include both negative-energy and positive-energy modes. In particular, both cases of wave propagation parallel and antiparallel to the electron beam are treated. In conventional free-electron lasers and wave collective ion accelerators, the attention is usually directed toward the negativeenergy mode and wave propagation in the beam direction. However, it was shown recently ${ }^{3}$ that the positiveenergy space-charge wave traveling in counter direction to the electron beam can also give rise to downconversion free-electron laser instabilities.

First, we review briefly the basic properties of the linear dispersion relations of space-charge waves in a relativistic electron beam given by ${ }^{12} \omega-v_{b} k= \pm \omega_{p} / \gamma_{b}^{3 / 2}$, where $v_{b}$ is the equilibrium electron beam velocity and

$$
\gamma_{b}=\left(1-v_{b}^{2} / c^{2}\right)^{-1 / 2} \text {. }
$$

It follows that the wave energy density for the spacecharge waves ${ }^{13}$ is proportional to $\omega /\left(\omega-v_{b} k\right)^{3}$. Hence, for the negative-energy (or slow) mode

$$
\omega-v_{b} k=\mp \omega_{p} / \gamma_{b}^{3 / 2},
$$

where the minus (plus) signs refer to $\omega>0(\omega<0)$, and for the positive-energy (or fast) mode

$$
\omega-v_{b} k= \pm \omega_{p} / \gamma_{b}^{3 / 2} \text {, }
$$

where plus (minus) refer to $\omega>0(\omega<0)$. From Eq. (1) we see that the phase velocity of the negative-energy mode is

$$
v_{\mathrm{ph}}=v_{b}\left(\frac{\omega}{\omega \pm \omega_{p} / \gamma_{b}^{3 / 2}}\right),
$$

where plus (minus) refer to $\omega>0(\omega<0)$, and the phase velocity of the positive-energy mode is

$$
v_{\mathrm{ph}}=v_{b}\left(\frac{\omega}{\omega \mp \omega_{p} / \gamma_{b}^{3 / 2}}\right) \text {, }
$$

where minus (plus) refer to $\omega>0 \quad(\omega<0)$. For the negative-energy mode $0<v_{\mathrm{ph}}<v_{b}$ for all $\omega$, whereas for the positive-energy mode $v_{b}<v_{\mathrm{ph}}<+\infty$ if $|\omega|>\omega_{p} / \gamma_{b}^{3 / 2}$ and $-\infty<v_{\mathrm{ph}}<0$ if $|\omega|<\omega_{p} / \gamma_{b}^{3 / 2}$. Hence it is evident that the negative-energy mode always propagates in the beam direction, whereas the positive-energy mode propagates in the direction opposite to the beam if $|\omega|<\omega_{p} / \gamma_{b}^{3 / 2}$.

Before carrying out a rigorous derivation of the nonlinear dispersion relation for space-charge waves, we shall first give a heuristic derivation that indicates the relation between a stationary electron plasma and a relativistic electron beam. In the beam (primed) frame in which $v_{b}^{\prime}=0$, the amplitude-dependent frequency of relativistic 
nonlinear electron plasma waves in a cold plasma is independent of $k^{\prime}$ and is approximately given by ${ }^{8,9}$

$$
\omega^{\prime} \simeq \pm \omega_{p}^{\prime} / \gamma_{0}^{\prime 1 / 2} \equiv \pm \Omega_{p}^{\prime}
$$

where $\omega_{p}^{\prime}=\left(n_{0}^{\prime} e^{2} / m \epsilon_{0}\right)^{1 / 2}, \gamma_{0}^{\prime}=\left(1-v_{0}^{\prime 2} / c^{2}\right)^{-1 / 2}$, and $v_{0}^{\prime}$ is the peak electron quiver velocity. Thus the wave oscillates at the proper plasma frequency $\Omega_{p}^{\prime}$ with the electron rest mass $m$ replaced by the relativistically corrected mass $\gamma_{0} m$. A Lorentz transformation of Eq. (3) to the laboratory frame (where the beam has a velocity $v_{b}$ ), using the relations $\omega^{\prime}=\gamma_{b}\left(\omega-v_{b} k\right)$ and $\Omega_{p}^{\prime}=\Omega_{p} / \gamma_{b}^{1 / 2}$, yields the following nonlinear dispersion relation for space-charge waves in a relativistic electron beam:

$$
\omega-v_{b} k= \pm \Omega_{p} / \gamma_{b}^{3 / 2},
$$

where the amplitude-dependent plasma frequency

$$
\Omega_{p}=\left(n_{0} e^{2} / \gamma_{0}^{\prime} m \epsilon_{0}\right)^{1 / 2}
$$

and

$$
\gamma_{0}^{\prime}=\gamma_{b}\left(\gamma_{0}-v_{b} \gamma_{0} v_{0} / c^{2}\right)
$$

In the linear limit ( $\left.\gamma_{0}^{\prime}=1\right)$, Eq. (4) reduces to Eq. (1).

Now, we derive the exact nonlinear dispersion relation of space-charge waves in a relativistic electron beam. The relativistic momentum and continuity equations, together with Poisson's equation, describe the cold electron beam

$$
\begin{aligned}
& \frac{\partial p}{\partial t}+v \frac{\partial}{\partial x} p=-e E \\
& \frac{\partial n}{\partial t}+\frac{\partial}{\partial x}(n v)=0, \\
& \epsilon_{0} \frac{\partial E}{\partial x}=e\left(n_{0}-n\right),
\end{aligned}
$$

where $p=m \gamma v, \gamma=\left(1-v^{2} / c^{2}\right)^{-1 / 2}$, and one-dimensional fluid is considered since the wave motion of space-charge waves is longitudinal. Seeking periodic wave solutions which depend only on the variable $\tau=\omega_{p}\left(t-x / v_{\mathrm{ph}}\right)$, we obtain from Eq. (6) the following equation for the electron-beam density:

$$
\frac{n}{n_{0}}=\frac{v_{\mathrm{ph}}-v_{b}}{v_{\mathrm{ph}}-v},
$$

where the constant of integration is chosen so that, $v_{b} \equiv\langle n v\rangle /\langle n\rangle$ and $n_{0} \equiv\langle n\rangle$, where \langle\rangle denotes averaging over a period in $\tau$. Note that, in the nonlinear theory, the equilibrium value of the electron-beam velocity $v_{b}$ has to be defined self-consistently. 9,14 The particular choice of integration constant in Eq. (8) allows us to study perturbations in the electron-beam density, which differs from the previous works on the plasma wake-field accelerator ${ }^{10,11}$ wherein the beam density perturbation is neglected. Taking the $\tau$ derivative of the momentum equation and substituting Poisson's equation and Eq. (8), we arrive at the following nonlinear wave equation:

$$
\frac{d^{2}}{d \tau^{2}}\left(\gamma-\beta_{\mathrm{ph}} u\right)=\beta_{\mathrm{ph}}^{2}\left(\frac{\beta-\beta_{b}}{\beta_{\mathrm{ph}}-\beta}\right),
$$

where $u=\gamma \beta, \beta=v / c, \beta_{\mathrm{ph}}=v_{\mathrm{ph}} / c$, and $\beta_{b}=v_{b} / c$. The first integral of Eq. (9) is

$$
\frac{1}{2}\left(\frac{d u}{d \tau}\right)^{2}=\frac{W-\gamma+\beta_{b} u}{\left(1-\beta / \beta_{\mathrm{ph}}\right)^{2}},
$$

where $W$ is an arbitrary constant of integration, greater than $1 / \gamma_{b}$, that determines the amplitude of wave oscillations. A further integration of Eq. (10) gives

$$
\tau= \pm \frac{\sqrt{2}}{2} \int_{0}^{u} \frac{1-\beta / \beta_{\mathrm{ph}}}{\left(W-\gamma+\beta_{b} u\right)^{1 / 2}} d u \text {. }
$$

Analysis of Eqs. (10) and (11) shows that $u$ is a periodic function of $\tau$. The turning points (i.e., $d u / d \tau=0$ ) are determined by setting $W-\gamma+\beta_{0} u=0$; thus the maximum and minimum of $u$ are

$$
u_{1,2}=\gamma_{b}^{2}\left[\beta_{b} W \pm\left(W^{2}-1 / \gamma_{b}^{2}\right)^{1 / 2}\right] .
$$

The period of oscillation is given by

$$
P= \pm \frac{\sqrt{2}}{\omega_{p}} \int_{u_{1}}^{u_{2}} \frac{1-\beta / \beta_{\mathrm{ph}}}{\left(W-\gamma+\beta_{b} u\right)^{1 / 2}} d u \text {. }
$$

Writing in terms of the frequency $\omega=2 \pi / P$, we then obtain the following exact nonlinear dispersion relation of space-charge waves in a relativistic electron beam:

$$
\omega= \pm \frac{\sqrt{2} \pi \omega_{p}}{\int_{u_{1}}^{u_{2}} \frac{1-\beta / \beta_{\mathrm{ph}}}{\left(W-\gamma+\beta_{b} u\right)^{1 / 2}} d u}
$$

which can be rewritten in a form analogous to Eqs. (1) and (4) as

$\omega-v_{b} k= \pm \frac{\sqrt{2} \pi \omega_{p}}{\int_{u_{1}}^{u_{2}} \frac{\beta_{\mathrm{ph}}-\beta}{\left(\beta_{\mathrm{ph}}-\beta_{b}\right)\left(W-\gamma+\beta_{b} u\right)^{1 / 2}} d u}$.

where the plus (minus) signs refer, respectively, to the positive- (negative-) energy mode if $\omega>0$ and the reverse if $\omega<0$.

Equations (12) and (13) admit both superluminous $\left(v_{\mathrm{ph}}>c\right)$ and subluminous $\left(v_{\mathrm{ph}}<c\right)$ space-charge waves. Wave breaking does not occur for superluminous waves since in that case the electron velocity may never reach the wave phase velocity. However, for subluminous waves there is the possibility of wave breaking.

In the beam frame, the electron density is ${ }^{8,9}$

$$
n^{\prime}=\frac{v_{\mathrm{ph}}^{\prime} n_{0}^{\prime}}{v_{\mathrm{ph}}^{\prime}-v^{\prime}},
$$

which combined with the fact that $v^{\prime}$ oscillates between the turning points $\pm v_{0}^{\prime}$ shows that wave solutions exist provided

$$
-v_{\mathrm{ph}}^{\prime}<v^{\prime}<v_{\mathrm{ph}}^{\prime} \text {. }
$$

In the laboratory frame, it is evident from Eq. (8) that wave solutions for space-charge waves are bounded by 


$$
\begin{aligned}
& v<v_{\mathrm{ph}} \text { if } v_{\mathrm{ph}}>v_{b}, \\
& v>v_{\mathrm{ph}} \text { if } v_{\mathrm{ph}}<v_{b} .
\end{aligned}
$$

Equation (16) corresponds to the upper bound of Eq. (15) in the beam frame, namely, $v^{\prime}=v_{\mathrm{ph}}^{\prime}$. Another limitation on the electron velocity can be obtained by Lorentz transforming the lower bound of Eq. (15), $v^{\prime}=-v_{\mathrm{ph}}^{\prime}$, to the laboratory frame using the relation

$$
v^{\prime}=\left(v-v_{b}\right) /\left(1-v_{b} v / c^{2}\right),
$$

which gives

$$
v=\frac{2 v_{b}-v_{\mathrm{ph}}-\beta_{b}^{2} v_{\mathrm{ph}}}{1+\beta_{b}^{2}-2 \beta_{b} \beta_{\mathrm{ph}}} \equiv v_{\mathrm{cr}} .
$$

Hence the existence condition for space-charge waves in a relativistic electron beam, valid for both negativeenergy and positive-energy modes, is

$$
\begin{gathered}
v_{\mathrm{cr}}<v<v_{\mathrm{ph}} \text { if } v_{\mathrm{ph}}>v_{b}, \\
v_{\mathrm{ph}}<v<v_{\mathrm{cr}} \text { if } v_{\mathrm{ph}}<v_{b} .
\end{gathered}
$$

Violation of Eq. (18) implies wave breaking. A graphical display of the condition (18) is presented in Fig. 1.

Next, we derive an expression for the wave-breaking amplitude of space-charge waves. It follows, from Eqs. (5) and (10), that the electric field inside the beam is

$$
E= \pm \sqrt{2}\left(m \omega_{p} c / e\right)\left(W-\gamma+\beta_{b} u\right)^{1 / 2} .
$$

Equation (19) shows that the maximum of $E$ (i.e., $d E / d u=0$ ) is given by $v=v_{b}$, thus

$$
E_{\max }=\sqrt{2}\left(m \omega_{p} c / e\right)\left(W-1 / \gamma_{b}\right)^{1 / 2} .
$$

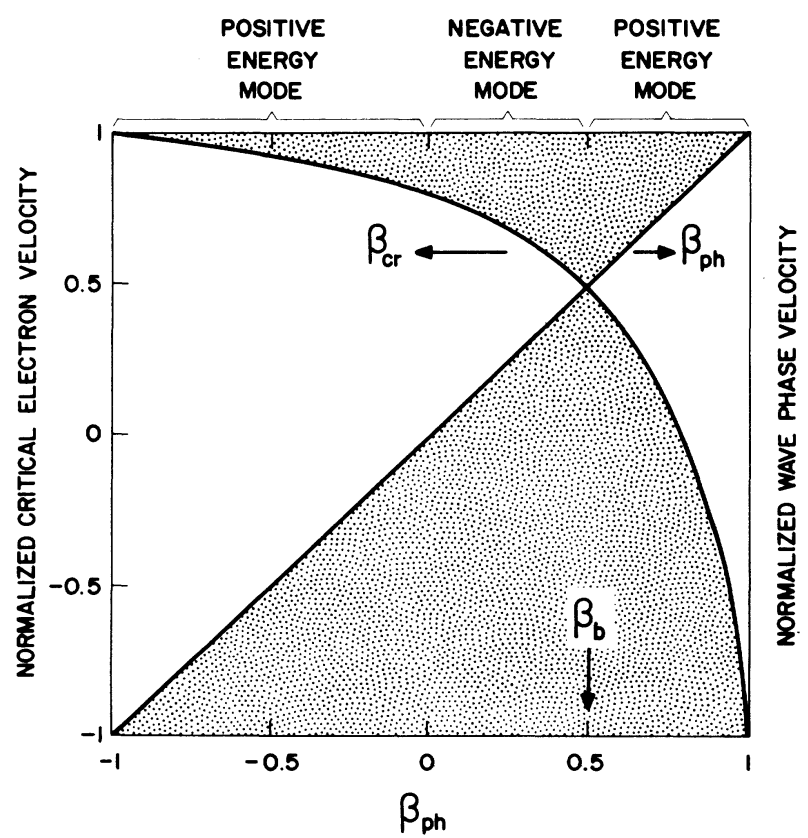

FIG. 1. Plot of the upper and lower bounds of the electron velocity as a function of $\beta_{\mathrm{ph}}$ for $\beta_{b}=0.5, \beta_{\mathrm{cr}}=v_{\mathrm{cr}} / c$. Shaded region indicates wave breaking.

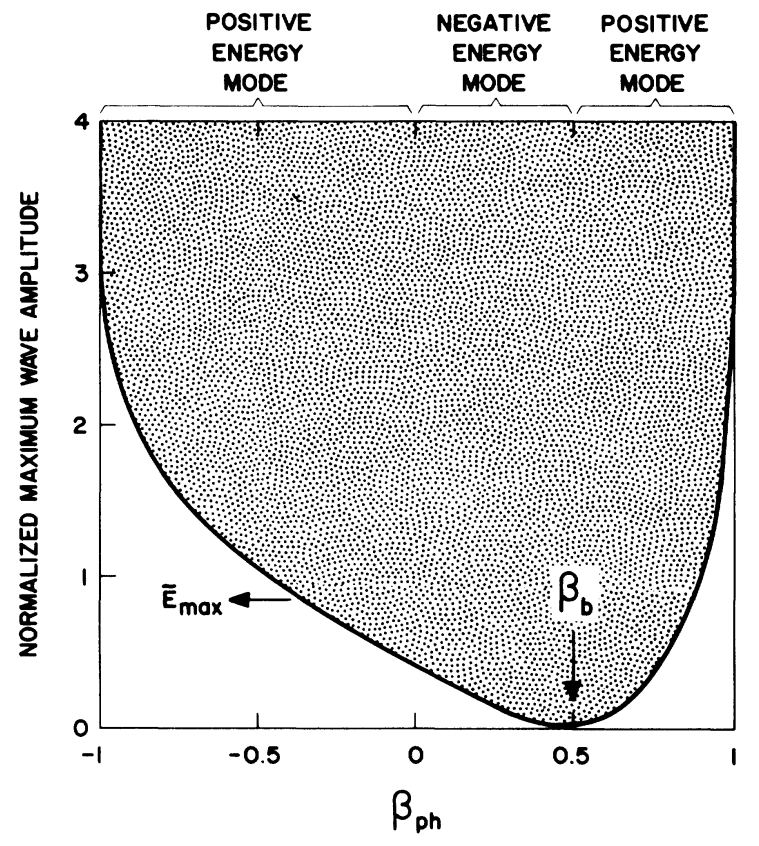

FIG. 2. Plot of the normalized maximum wave amplitude $\widetilde{E}_{\max }=e E_{\max } / m \omega_{p} c$ as a function of $\beta_{\mathrm{ph}}$ for $\beta_{b}=0.5$. Shaded region indicates wave breaking.

Now, $W$ is related to the turning points of $u$ through the equation $W-\gamma+\beta_{b} u=0$, and according to Eq. (18) the critical turning points for which wave breaking occurs are given by $v=v_{\mathrm{cr}}$ or $v=v_{\mathrm{ph}}$. Hence, at the onset of wave breaking, the value $W$ is

$$
W=\gamma_{\mathrm{cr}}-\beta_{b} u_{\mathrm{cr}}=\gamma_{\mathrm{ph}}-\beta_{b} u_{\mathrm{ph}} .
$$

The final expression for the maximum electric field is obtained by substituting Eq. (21) in Eq. (20), yielding

$E_{\max }=\sqrt{2}\left(m \omega_{p} c / e\right)\left[\gamma_{\mathrm{ph}}\left(1-\beta_{\mathrm{ph}} \beta_{b}\right)-1 / \gamma_{b}\right]^{1 / 2}$.

This expression agrees with a Lorentz transformation of the expression for wave breaking in a stationary plasma. Equation (22), valid for both negative-energy and positive-energy space-charge waves, determines the critical wave amplitude above which wave breaking takes place. A graphical display of Eq. (22) is shown in Fig. 2. It is expected that the effect of beam temperature can reduce the wave-breaking amplitude. ${ }^{11,15,16}$

Equations (18) and (22) provide alternative means for analyzing the wave-breaking condition of space-charge waves in a relativistic electron beam. It can be concluded, with the aid of Figs. 1 and 2, that for a given $v_{b}$ : (i) for either the negative-energy or positive-energy modes, $E_{\max } \rightarrow 0$ as $v_{\mathrm{ph}} \rightarrow v_{b}$; (ii) for the negative-energy mode $E_{\max }$ decreases as $v_{\mathrm{ph}}$ increases, and (iii) for the positiveenergy mode, $E_{\max }$ increases as $v_{\mathrm{ph}}$ departs from $v_{\mathrm{b}}$ and $E_{\max } \rightarrow \infty$ as $\left|v_{\mathrm{ph}}\right| \rightarrow c$.

The breaking of space-charge waves studied in this paper is of fundamental relevance for devices driven by intense electron beams. For example, it determines the largest accelerating field obtainable in wave collective ion 
accelerators $^{4-6}$ as well as wake-field accelerators, ${ }^{10,11,17}$ and may be a nonlinear saturation mechanism for freeelectron lasers. ${ }^{1-3,18}$ Our results indicate that wave breaking imposes severe limitation on devices that employ space-charge waves, either negative-energy or positive-energy modes, with phase velocity close to the electron-beam velocity since in that case the normalized maximum wave amplitude $e E_{\max } / m \omega_{p} c \ll 1$. On the other hand, wave breaking presents little (or no) restriction for devices that employ positive-energy space-charge waves, either parallel or antiparallel propagating, with phase speed near (or greater than) the speed of light, since in that case extremely large wave amplitudes, such that $e E_{\max } / m \omega_{p} c \gg 1$, are attainable.

It is important to note that the phenomenon of particle trapping can compete with wave breaking to limit the amplitude of space-charge waves. If the wave amplitude is sufficiently large, a substantial fraction of beam electrons can become trapped in the wave potential well. In the reference frame moving with the wave phase velocity, the condition for the onset of particle trapping ${ }^{18}$ is $(\gamma-1) m c^{2} \lesssim\left|e \phi_{\max }\right|$, namely, when the electrostatic potential energy of space-charge waves exceeds the kinetic energy of beam particles. When this occurs, particle trapping may limit the wave amplitude before wave breaking takes place. For example, according to Eq. (22), the wave-breaking amplitude becomes infinite in the limit $\left|v_{\mathrm{ph}}\right|=c$. Physically, however, when the wave amplitude satisfies the aforementioned condition, electrons become trapped and continuously gain energy from the wave, thus limiting the wave amplitude, even though wave breaking has not occurred mathematically. On the other hand, if the wave amplitude is too small to trap electrons, then wave breaking is the physical mechanism that determines the maximum amplitude attainable by spacecharge waves. Equation (8) shows that wave breaking is the consequence of wave profile steepening as the electron velocity approaches the wave phase velocity. Hence, in terms of Eq. (8), the mathematical condition for the onset of wave breaking is $v=v_{\mathrm{ph}}$. At the critical condition $v=v_{\mathrm{ph}}$, both $n$ and $\partial E / \partial x$ become infinite. When $v>v_{\mathrm{ph}}$, the wave field becomes multivalued and the wave profile begins to break. After wave breaking occurs, the wave energy is transferred to beam particles, leading to the generation of highly energetic electrons. Physically, the cause of wave breaking can be attributed to redistribution of the wave energy spectrum through nonlinear effects. A perturbation analysis ${ }^{9}$ of the nonlinear wave equation shows that as the wave amplitude increases the energy is fed into wave components with higher and higher frequencies and wave numbers. This leads to the change of wave profile and eventually results in the breaking of the wave.

\section{ACKNOWLEDGMENTS}

The author would like to thank J. J. Barroso for a critical reading of the manuscript.
${ }^{1}$ T. Kwan, J. M. Dawson, and A. T. Lin, Phys. Fluids 20, 581 (1977).

${ }^{2}$ T. C. Marshall, Free-Electron Lasers (Macmillan, New York, 1985).

${ }^{3}$ B. Steinberg, A. Gover, and S. Ruschin, Phys. Rev. A 33, 421 (1986).

${ }^{4}$ P. Sprangle, A. T. Drobot, and W. M. Manheimer, Phys. Rev. Lett. 36, 1180 (1976).

${ }^{5}$ S. V. Yadavalli, Appl. Phys. Lett. 29, 272 (1976).

${ }^{6}$ G. Gammel, J. A. Nation, and M. E. Read, J. Appl. Phys. 50, 5603 (1979).

${ }^{7}$ J. M. Dawson, Phys. Rev. 113, 383 (1959).

${ }^{8}$ A. I. Akheizer and R. V. Polovin, Zh. Eksp. Teor. Fiz. 30, 915 (1956) [Sov. Phys._JETP 3, 696 (1956)].
${ }^{9}$ A. C. -L. Chian, Plasma Phys. 21, 509 (1979).

${ }^{10}$ J. B. Rosenzweig, Phys. Rev. Lett. 58, 555 (1987).

${ }^{11}$ T. Katsouleas and W. B. Mori, Phys. Rev. Lett. 61, 90 (1988).

${ }^{12}$ P. C. Clemmow and J. P. Dougherty, Electrodynamics of Particles and Plasmas (Addison-Wesley, Reading, MA, 1969), p. 205.

${ }^{13} \mathrm{~A}$. Hasagawa, Plasma Instabilities and Nonlinear Effects (Springer-Verlag, Berlin, 1975), p. 17.

${ }^{14}$ A. C. - L. Chian, Phys. Rev. A 24, 2773 (1981).

${ }^{15}$ T. P. Coffey, Phys. Fluids 14, 1402 (1971).

${ }^{16}$ W. Kruer, Phys. Fluids 22, 1111 (1979).

${ }^{17}$ Y. T. Yan, Phys. Fluids 31, 960 (1988).

${ }^{18}$ A. T. Lin and J. M. Dawson, Phys. Fluids 23, 1224 (1980). 